\title{
Salicylic Acid Induced Physiological Responses of Microchaete Sp. NCCU-342 Under PQ Stress
}

\author{
Haleema Naaz \\ Jamia Millia Islamia \\ Bushra Afzal \\ Jamia Millia Islamia \\ Neha Sami \\ Jamia Millia Islamia \\ Durdana Yasin \\ Jamia Millia Islamia \\ Nida Jamil Khan \\ Jamia Millia Islamia \\ Tasneem Fatma ( $\square$ fatma_cbl@yahoo.com ) \\ Jamia Millia Islamia
}

\section{Research Article}

Keywords: Weed, Bio-fertilizer, Herbicide, Glutathione, Microchaete sp. NCCU-342

Posted Date: August 30th, 2021

DOl: https://doi.org/10.21203/rs.3.rs-839759/v1

License: (c) (i) This work is licensed under a Creative Commons Attribution 4.0 International License.

Read Full License 


\section{Abstract}

Weeds are the worst category of agricultural pests as they compete with crops for water, nutrients, and light, grow faster than crops and eventually reduces the crop productivity thereby increasing the production cost. Herbicide has been used as a management tool to control weeds, by modifying their physiological activities like reducing photosynthesis, increasing protease activity and free radicals etc. The application of non-selected herbicides kills not only the weeds but also non-target organisms including the main crop. Herbicides like paraquat have been used in rice fields but its toxic effects on other organisms is at par. Salicylic acid, a plant growth regulator, is known to regulate oxidative stress in plants subjected to unfavourable environmental conditions. Given this, the present study was designed to study the effect of salicylic acid in Microchaete sp. NCCU-342 exposed to paraquat. The results obtained demonstrated that the paraquat toxicity elevated MDA and $\mathrm{H}_{2} \mathrm{O}_{2}$ levels, indicating free radical formation. However, supplementation of salicylic acid in the culture medium reduced the paraquat toxicity. The content of MDA and $\mathrm{H}_{2} \mathrm{O}_{2}$ in presence of salicylic acid was similar to the control. It enhances phenolic compound (29.07\%) and phytohormones content (IAA-2.96\%, SA-96.59\%) and regulate the redox state of the cells. A significant change (recorded in percentage) was observed in the antioxidant enzyme activities including SOD (121.62 \%), CAT (30.38\%), APX (74.38 \%), GPX (127\%) and GR (141\%) in presence of paraquat and after supplementation of salicylic acid. Antioxidant assay confirmed the change in antioxidants in form of phenolic compounds. Salicylic acid supplementation also enhanced the antibacterial activity of cyanobacteria, highlighting that it induces resistance against biotic stress as well as the abiotic stress. This work provides evidence for the ability of salicylic acid to alleviate the paraquatinduced toxicity, maintain redox state and resist the cell against both biotic and abiotic stress.

\section{Introduction}

Herbicide through increase crop yield but their residual toxic impact on both selective and non-selective target, in long run disrupts the ecological balance between soil, crop and microflora ${ }^{1}$. Paraquat (PQ) is a very well-known non-selective herbicide that has been widely used in agriculture fields such as Wheat, Rice, Maize, Cotton, Potato and has shown high toxicity against plants, animals and humans ${ }^{2}$. PQ acts by reducing the production of NADPH in a photosynthetic organism. It is an oxidant that interferes with the electron transfer reaction by accepting the electron from photosystem I and transferring it to molecular oxygen and producing reactive oxygen species (ROS) and reducing the formation of NADPH ${ }^{3}$. Accumulation of ROS leads to lipid peroxidation and induces membrane damage, breakdown of photosynthetic apparatus, ion leakage, eventually leading to cell death and overall reduction in the crop $y^{\text {yield }}{ }^{4}$. The antioxidant enzymes are known as scavenger of ROS and their expression facilitates plant survival under stress conditions. Superoxide dismutase (SOD) is known to scavenge superoxide radicals, whereas catalase (CAT) and ascorbate peroxidase (APX) function as a scavenger of hydrogen peroxide $\left(\mathrm{H}_{2} \mathrm{O}_{2}\right)$. Herbicide toxicity alters activities of these enzymes by activating protease and the plants succumb to the unfavourable conditions ${ }^{5}$. 
Cyanobacteria are the pioneer photosynthetic bacteria that are known to play an important role in sustainable agriculture in rice ecosystem. Nostoc muscorum Anabaena sp., Calothrix, Microchaete sp. are classified as biofertilizers as they fix nitrogen, release plant growth promoter substances such as phenols, phytohormones, and increase the water holding capacity of soil ${ }^{6}$. Application of $P Q$ in rice field is known to effect cyanobacteria that is used as biofertilizer ${ }^{7}$.

Salicylic acid (SA) is an important signalling molecule released by plants under stressful conditions ${ }^{8}$. It is an endogenous plant hormone that modulates metabolic and physiologic response under oxidative stress $^{9}$ as induced by salinity ${ }^{10}$, heavy metal ${ }^{11}$, drought ${ }^{12}$, heat ${ }^{13}$, cold $^{14}$ and herbicide ${ }^{3}$. It has been reported that exogenous application of salicylic acid also reduces the toxicity of herbicide by modifying the antioxidant enzyme activity ${ }^{3}$. SA is known to control the pesticide effect such as H.A.M-45, Termite kill, Anchor in Green gram ${ }^{15}$, Napropamide in rapeseed and mustard plants ${ }^{16}$ and Clethodium in maize ${ }^{17}$. In barley plant under paraquat toxicity, the treatment of salicylic acid-induced the expression of SOD, CAT, $\mathrm{APX}$, and $\mathrm{POD}^{3}$. Exogenous application of $\mathrm{SA}$ is known to reduce the quinclorac (herbicide) toxicity by modulating the antioxidant defense enzymes such as SOD, CAT, APX, GPX and GR ${ }^{18}$. SA also elevates the content of non-enzymatic antioxidants such as ascorbate and glutathione which are directly involved in the detoxification of ROS and also play an active role in ascorbate-glutathione cycle to maintain the redox state $^{19}$. Glutathione performed the redox couple reaction with nicotinamide adenine dinucleotide phosphate and provides stress resistance to the plants ${ }^{20}$. Supplementation of SA in wheat seedlings showed the increased content of ascorbate, glutathione and other related enzymes ${ }^{21}$.

To validate our hypothesis that SA helps in maintaining the cellular redox state of cyanobacteria under $\mathrm{PQ}$ stress. "Cyanobacteria culture treated with PQ was grown in presence and absence of SA for 96 hours. The endogenous phytohormones (IAA and SA), phenolic content, redox biomarker (GSH and GSSH and its ratio), antioxidant enzymes, DPPH (for free radical scavenging potential) and anti-bacterial activity (for biotic potential) were evaluated as these parameters reflect the tolerant potential of the organism.

\section{Materials And Methods}

Culture maintenance. Microchaete sp. NCCU-342 were procured from IARI, New Delhi and grown in BG-11 medium without sodium nitrate illuminated with the fluorescent light with $2000 \pm 200$ lux intensity for 12:12 light/dark cycle under the aseptic culture room ${ }^{54}$.

Growth of Microchaete sp. NCCU-342 in presence of PQ and SA. Microchaete sp. NCCU-342 was grown in presence of $P Q(0,0.1,0.2,0.4,0.6,0.8,1.0,1.2 \mu \mathrm{M})$ to determine its tolerance limit. After 96 hours, biomass was washed twice with distilled water, harvested by centrifugation (5000 rpm for 10 minutes) and dried at $60^{\circ} \mathrm{C}$. Growth of the organism was check by the chlorophyll content. Total chlorophyll was analyzed according to the modified protocol of Mackinney, (1942) ${ }^{55}$. Dried biomass was dissolved in 95 $\%$ methanol $(10 \mathrm{ml})$ and incubated at $65^{\circ} \mathrm{C}$ for 30 minutes in a water bath, centrifuged at $5000 \mathrm{rpm}$ for 
10 minutes and absorbance of the supernatant was taken at $650 \mathrm{~nm}$ and $660 \mathrm{~nm}$ against $95 \%$ methanol as blank. Total chlorophyll was calculated by using the following formula.

$$
\text { Chlorophyllcontent }(\mathrm{mg} / \mathrm{g})=\left(2.55 \times O D_{650 \mathrm{~nm}}+0.4 \times O D_{665 \mathrm{~nm}}\right) \times 10^{-2}
$$

Tolerance limit of $P Q$ was determined through linear regression 56 . Further, at $50 \%$ inhibitory concentration, $0.3 \mathrm{mM}$ of SA was supplemented to check the physiological parameters i.e., endogenous phytohormones (IAA and SA), phenolic content, redox biomarkers (GSH and GSSH and its ratio), antioxidant enzymes, DPPH (for free radical scavenging potential) and anti-bacterial activity (for biotic potential).

Determination of Malondialdehyde and hydrogen peroxide (supplementary data). Malondialdehyde (MDA) and hydrogen peroxide was estimated as done in our previous study ${ }^{57}$.

Determination of total Phenol content. The total phenolic contents were estimated by using protocol of Singleton and Rossi (1965) ${ }^{58}$. Dried biomass (50 mg) was homogenized in $80 \%$ methanol $(10 \mathrm{ml})$. Distilled water $(5 \mathrm{ml})$ and Folin Ciocalteu's reagent $(0.5 \mathrm{ml})$ were added to the methanolic extract $(40 \mu \mathrm{L})$ and kept for 5 minutes. Then, $20 \%$ sodium carbonate $(1.2 \mathrm{ml})$ was added and the volume of the test sample was adjusted to $10 \mathrm{ml}$ with distilled water and allowed to stand in dark at room temperature for 60 minutes. Absorbance was recorded at $750 \mathrm{~nm}$ after the development of intense blue colour. This equation was used to calculate the amount of standard gallic acid with the regression coefficient $\left(\mathrm{r}^{2}\right)$ being 0.9666 .

$$
Y=0.0018 \times X+0.0132
$$

Determination of endogenous indole acetic acid (IAA) and salicylic acid (SA) content. The protocol of Ehmann (1977) ${ }^{59}$ was used to determine the IAA content produced. A colorimetric technique was adopted by using Salkowski reagent (one $\mathrm{ml}$ of $50 \mathrm{mM}$ ferric chloride dissolved in $35 \%$ perchloric acid). Biomass (50 mg) was centrifuged at 10,000 rpm for 15 minutes at $4^{\circ} \mathrm{C}$. The supernatant was collected and filtered by Millipore filter, $0.45 \mu \mathrm{m}$ and acidified with $1.0 \mathrm{M}$ hydrochloric acid. Extraction was done by partitioning with ethyl acetate (1:3, v/v; thirce) and evaporated using Rotary evaporator (Buchi type). Dried extract was dissolved in $95 \%$ methanol $(1 \mathrm{ml})$. Salkowaski reagent $(1 \mathrm{ml})$ was added to this extracted methanolic solution $(0.5 \mathrm{ml})$ and kept in dark. Development of the red-pink colour indicates the presence of IAA and the absorabnce was taken at $530 \mathrm{~nm}$, against Salkowski reagent as blank. Standard IAA (1 mg/ ml) was prepared as stock solution. It was further diluted with distilled water to obtain different working concentrations $(0.5-5 \mu \mathrm{g} / \mathrm{ml})$. The calibration curve was described by the equation given below with the regression coefficient $\left(r^{2}\right)$ being 0.9779 . This equation was used to calculate the amount of standard IAA.

$$
Y=0.1423 \times X+0.0035
$$

Endogenous salicylic acid production was also checked. Salicylic acid extraction was done by using the protocol of Raskin et al. (1989) ${ }^{60}$ and quantification was done by following the protocol of Warrier et al. 
$(2013)^{61}$. For extraction of endogenous salicylic acid, $50 \mathrm{mg}$ biomass was homogenized with $90 \%$ methanol $(10 \mathrm{ml})$ and centrifuged at $5000 \mathrm{rpm}$. Pellet was dissolved in absolute methanol $(10 \mathrm{ml})$ and centrifuged at $8000 \mathrm{rpm}$ for 15 minutes. The supernatants were evaporated at $60^{\circ} \mathrm{C}$ and the residue was re-suspended in $5 \%$ trichloroacetic acid $(5 \mathrm{ml})$ and centrifuged at $5000 \mathrm{rpm}$. The supernatant was partitioned twice with double the volume of ethyl acetate ${ }^{60}$. The production of salicylic acid was confirmed by simple colorimetric test. To the ethyl extract $(1 \mathrm{ml})$, alcoholic ferric chloride $(1 \mathrm{ml})$ was added, formation of purple-violet colour indicated the presence of salicylic acid that was measured at 540 $\mathrm{nm}$. Salicylic acid stock solution $(1 \mathrm{mg} / \mathrm{ml})$ was further diluted with distilled water to obtain different working concentrations $(0.01-0.5 \mu \mathrm{g} / \mathrm{ml})$. Absorbance was taken at $540 \mathrm{~nm}$. The calibration curve was described by the equation given below with regression co-efficient $\left(r^{2}\right)$ being 0.9582 . The following equation was used to calculate the amount of standard salicylic acid.

$$
Y=1.9821 \times X+0.0148
$$

Where $Y$ is absorbance at $540 \mathrm{~nm}$ and $X$ is concentration of salicylic acid in $\mu \mathrm{g} / \mathrm{ml}$.

Determination of reduced and oxidized glutathione (GSH) and redox state. Glutathione was determined by the method of Anderson, $(1985)^{62}$. Fresh biomass (50 mg) was homogenized in $5 \%$ sulfosalicylic acid and centrifuged at 10,000 rpm for 10 minutes under cold condition. After centrifugation, in $500 \mu \mathrm{l}$ supernatant was added to $600 \mu \mathrm{l}$ of phosphate buffer $(100 \mathrm{mM}, \mathrm{pH} 7.0)$ and DTNB (40 $\mu \mathrm{l})$ and incubate for 2 minutes at room temperature. After incubation absorbance was read at $412 \mathrm{~nm}$. For oxidized glutathione, the GSH was masked by derivatization with 2-vinylpyridine. Reduced glutathione was calculated by subtracting the value of oxidized glutathione from the total glutathione. The redox state was calculated as the ratio of GSH to oxidized glutathione (GSSG).

\section{Determination of Superoxide dismutase (SOD), Catalase (CAT) and Ascorbate peroxidase (APX) activity.}

SOD, CAT and APX activity was estimated by the method as followed in our previous study 57

Determination of Glutathione Reductase (GR) activity. GR activity was estimated by the method of Asgher et al., (2014) ${ }^{63}$. Fresh biomass $(50 \mathrm{mg})$ was homogenized in phosphate buffer $(50 \mathrm{mM}, \mathrm{pH} 7.5)$ and centrifuged at $15,000 \mathrm{rpm}$ for 15 minutes at $4^{\circ} \mathrm{C}$. Reaction mixture $(3 \mathrm{ml})$ was prepared by mixing $0.5 \mathrm{mM}$ oxidized glutathione, $0.2 \mathrm{mM}$ NADPH in phosphate buffer $(25 \mathrm{mM}, \mathrm{pH} 7.8)$ and $100 \mu \mathrm{l}$ of supernatant. GR activity was determined by monitoring the glutathione-dependent oxidation of NADPH at $340 \mathrm{~nm}$ against blank of the same reaction mixture except for NADPH. $1 \mathrm{U}$ of the enzyme is the amount necessary to decompose $1 \mu \mathrm{l}$ of $\mathrm{NADPH}$ per minute at $25^{\circ} \mathrm{C}$ and GR activity was calculated by taking $\varepsilon_{340}$ as $6.2 \mathrm{mM}^{-}$ ${ }^{1} \mathrm{~cm}^{-1}$.

Determination of Glutathione peroxidase (GPX) activity. GPX activity was estimated by the method of Elia et al., (2003) ${ }^{64}$. Fresh biomass (50 mg) was homogenized in $50 \mathrm{mM}$ phosphate buffer (10 ml) having pH 7.5 and centrifuged at $15,000 \mathrm{rpm}$ for 15 minutes at $4^{\circ} \mathrm{C}$. Reaction mixture $(3 \mathrm{ml})$ was prepared by the mixing of $0.1 \mathrm{mM}$ EDTA, $0.1 \mathrm{mM} \mathrm{NADPH}, 0.1 \mathrm{mM} \mathrm{FeSO}_{4}, 0.1 \mathrm{mM} \mathrm{GSH}, 0.1 \mathrm{U}$ glutathione reductase, 0.1 
$\mathrm{mM}$ hydrogen peroxide in phosphate buffer $(50 \mathrm{mM}, \mathrm{pH} 7.0)$ and $100 \mu \mathrm{l}$ of supernatant. GPX Activity was determined by monitoring the glutathione-dependent oxidation of NADPH at $340 \mathrm{~nm}$ against blank of the same reaction mixture without NADPH. $1 \mathrm{U}$ of the enzyme is the amount necessary to decompose $1 \mu$ of hydrogen peroxide per minute at $25^{\circ} \mathrm{C}$ and GPX activity was calculated by taking $\varepsilon_{340}$ as $6.62 \mathrm{mM}^{-1} \mathrm{~cm}^{-1}$.

Determination of anti-oxidant activities. DPPH (2, 2-diphenyl-1-picrylhydrazyl) assay was performed according to the modified protocol of Shimada et al. (1992), ${ }^{65}$ in presence or absence of SA treatments under PQ stress to know the free radical scavenging potential of cells. Dried methanolic extract of each sample $(0,25,50,75,100,125,150, \mu \mathrm{g} / \mathrm{ml})$ was prepared. $3 \mathrm{ml}$ of DPPH solution $(0.1 \mathrm{mM}$ DPPH in $80 \%$ ethanol) was added in methanolic extract $(1 \mathrm{ml})$. Reaction mixture was shaken vigorously, left to stand in dark for 30 minutes and absorbance was recorded at $517 \mathrm{~nm}$. Percentage DPPH scavenging activity was calculated by using the following formula-

$$
D P P H S c a v e n g i n g a c t i v i t y=\frac{(A c-A t)}{A c} \times 100
$$

Determination of anti-bacterial activities. To know whether these treatments are inducing any resistance against bacteria, anti-bacterial activity of aqueous extracts of each culture was tested against Pseudomomas aeruginosa by using disc diffusion assay ${ }^{66}$. For which bacteria were streaked on Luria agar media plates and incubated at $37^{\circ} \mathrm{C}$ for overnight. After that, the pure single bacterial colony was inoculated in $10 \mathrm{ml}$ sterile Luria broth and incubated in a shaker at $37^{\circ} \mathrm{C}$ for 5 hours at $120 \mathrm{rpm}$ to obtain the log phase bacterial culture. According to $0.5 \mathrm{McF}$ arland $\left(1-2 \times 10^{8} \mathrm{CFU} / \mathrm{ml}\right)$, the absorbance of culture was adjusted to 0.1 at $600 \mathrm{~nm}$. Then, $50 \mu \mathrm{L}$ of bacterial culture was spread on Luria agar plates and 5 $\mathrm{mm}$ discs were placed at equal distance on each plate. Various concentrations of extracts $(20,40,60$ and $80 \mu \mathrm{g} / \mathrm{ml}$ ) from PQ treated biomass, SA treated, PQ and SA treated and control biomass (without any treatment) were taken for testing anti-bacterial activity. Streptomycin and water were used as positive and negative controls respectively. All the plates were overnight incubated at $37^{\circ} \mathrm{C}$. Zone of Inhibition (ZOI) was measured to determine their antibacterial potential.

Confocal laser scanning microscopy. Confocal microscopy was done by using Leica true confocal scanner used to check the effect of $\mathrm{PQ}$ on Microchaete sp. in presence and absence of salicylic acid ${ }^{67}$. In $1 \mathrm{ml}$ culture from all sets added in $2.5 \%(\mathrm{v} / \mathrm{v})$ glutaraldehyde phosphate buffer $(0.2 \mathrm{M}, \mathrm{pH} 7.0)$ for fixation and kept for 3 hours. Washed thrice with same buffer and stored at $4^{\circ} \mathrm{C}$. From this drop of sample was placed on slide and covered with glass slip slowly Slide was adjusted between stage clip and observed with a X63 1.4 numerical aperture and apochromat oil immersion objective lens under an excitation beam with a wavelength of $568 \mathrm{~nm}$ by an Ar-Kr laser beam.

\section{Concluding remarks}

Paraquat shows toxic effect to the non-target cyanobacterium, Microchaete sp. NCCU-342 by changing in their normal biochemical and metabolic activity. PQ increases oxidative damage through free radical 
formation. Supplementation of salicylic acid improves the antioxidant machinery, increases phenolic compound and phytohormones that ultimetly reduces the free radical level. Salicylic acid application also enhanced the antimicrobial activity of the organism. Supplementation of salicylic acid not only counteracts the toxic effect of herbicide through managing the antioxidant potential but also make it tolerant against biotic stress. Therefore, for the sustainable agriculture, the formulation of salicylic acid with cyanobacterial biofertilizer would be a better option.

\section{Results And Discussion}

During last few decades, treatment of herbicide has been adopted for better crop production. The nonselective herbicides like paraquat, glufosinate, basagran, and glyphosate not only affect the weed but also change the physiology of non-target organism like main crop and biofertilizer i.e., cyanobacteria ${ }^{22}$. $\mathrm{PQ}$ (methyl viologen) is a cationic herbicide that disturbs electron transport by accepting the electron from photosystem I. Cyanobacterial rice bio fertilizers like Microchaete $s p$. is also highly sensitive to PQ due to presence of photosystem I ${ }^{23}$. Microchaete $s p$. NCCU-342 is dominantly found in the rice field ecosystem as an important component that maintains the nitrogen status of soil to improve crop production. ${ }^{24}$

Effect of PQ and SA+PQ on the growth of Microchaete sp. NCCU-342. To understand the effect of PQ on Microchaete sp. NCCU-342, cultures were exposed to $0.1,0.2,0.4,0.6,0.8,1.0 \mu \mathrm{M}$ of PQ. Microchaete sp. NCCU-342 showed $86 \%, 74 \%, 64 \%, 50 \%, 24 \%, 19 \%$ growth reduction in presence of $0.1,0.2,0.4,0.6,0.8$, $1.0 \mu \mathrm{M}$ of $\mathrm{PQ}$, respectively as compared to control (Fig. 1a). The brownish green colour of cultures turned yellow to white and microscopic examination of culture showed cell lysis and depigmentation of cells. The reduction in growth under PQ stress have been also reported in other cyanobacterial strains such as Nostoc sp. N1 and Anabaena sp. A $1^{25}$, Anabaena variabilis and Plectonema boryanum ${ }^{26}$, Anabaena oryzae and Nostoc ellipsosporum ${ }^{27}$ and in microalgae like Chlorella vulgaris and Chlorella pyrenoidosa ${ }^{28}$.

For comparative growth analysis, Microchaete sp. were grown for 96 hours in presence of only PQ (0.6 $\mu \mathrm{M})$, and SA supplemented (0.3 mM) PQ exposed cultures. After 96 hours, the growth was found maximum in control. However, growth declined in $P Q$ treated culture (50\%) whereas supplementation of $S A$ in the $P Q$ treated culture showed higher growth (35\%) (significant at $P \leq 0.05$ ). Supplementation of exogenous SA in Microchaete sp. culture showed good growth than the PQ exposed cultures (Fig. 1b). The growth enhancement by the application of salicylic acid has been also reported in Chlorella vulgaris $^{29}$.

Chlorophylls represent the fundamental part of the whole metabolism of the photosynthetic organisms. Increase in chlorophyll content reflect growth, whereas decrease in chlorophyll reflect the unfavourable condition for growth. Under confocal microscopy, chlorophyll was used as a natural marker. Microchaete $s p$. NCCU-342 grown for 96 hours with $0.6 \mu \mathrm{M} \mathrm{PQ}$ exhibited degradation of chlorophyll. Normal healthy cells of control sample were more fluorescent that confirmed growth. PQ reduced the chlorophyll content 
and resulted in poor growth that decreased the fluorescent whereas supplementation of salicylic acid improved chlorophyll and reduced the membrane damage and lysis, and gave higher fluorescent (Fig. 1cf). Phormidium corallyticum under black band disease ${ }^{30}$ and in Synechococcus elongatus PCC 7942 also showed reduced fluorescence under hydrogen peroxide stress. ${ }^{31}$

Effect of $P Q$ and $S A+P Q$ on oxidative stress under $P Q$ stress. $P Q$ induced the production of free radicals MDA and hydrogen peroxide (Fig. 2a-b). MDA level increased by $8.16 \%$ in PQ exposed culture as compared to control and reduced by $15.63 \%$ in culture having both SA and PQ as compared to PQ treated culture (significant at $P \leq 0.05$ ). As compared to control, the level of hydrogen peroxide in $P Q$ treated set increased by $4.11 \%$ and reduction in hydrogen peroxide level was found as $2.455 \%$ in culture with both $\mathrm{SA}$ and $\mathrm{PQ}$ (significant at $\mathrm{P} \leq 0.05$ ). $\mathrm{PQ}$ (di-cation) disrupts the electron transport system and form the mono-radical cation. This mono-radical cation interacts with molecular oxygen to produce other toxic radical such as $02^{--}$and hydrogen peroxide ${ }^{32}$. According to Sood et al. $(2011)^{33}$, an increase in the concentration of $\mathrm{PQ}$ resulted in the generation of MDA and $\mathrm{H}_{2} \mathrm{O}_{2}$ in Azolla-Anabaena symbiotic system due to disruption of the cell membrane that loosens the integrity of the cell. Production of hydrogen peroxide is mainly due to the reduction of PQ di-cation by PSI that leads to the subsequent formation of various toxic radicals including hydrogen peroxide. Other herbicide like, glyphosate and bentazone increased the MDA and hydrogen peroxide content in Microcystis aeruginosa ${ }^{34}$ and Anabaena cylindrica ${ }^{35}$ SA was found to decrease oxidative damages and worked as a protective compound against $P Q$ induced toxicity. Application of $S A$ on $P Q$ treated culture reduced the lipid peroxidation and generation of hydrogen peroxide. Such observation have been also reported in higher plants e.g. barley under $\mathrm{PQ}^{36}$ maize under clethodium stress and Arachis hypogaea under Basagran stress ${ }^{37}$.

\section{Effect of $P Q$ and $S A+P Q$ on antioxidant enzymes under $P Q$ stress. The toxicity of $P Q$ causes the} generation of free radicals. In order to counteract these free radical's antioxidant enzymes like SOD acts as the first-line defense but produces hydrogen peroxide as its end product that gets eliminated by CAT, $\mathrm{APX}$ and GPX ${ }^{32,38}$. As compared to control, a $78.37 \%$ increase in SOD activity was recorded in $\mathrm{PQ}$ treated culture set. Supplementation of salicylic acid in PQ-treated culture was found to increase the activity by $121.62 \%$ as compared to control (Fig. 3a). Similarly, CAT (46.38\%), APX (16.73 \%), GPX (240 $\%)$ and GR (15\%) activity also increased in PQ treated culture as compared to control, but their induction was enhanced up to $30.38 \%$ in CAT, $74.38 \%$ in APX, $127 \%$ in GPX and $141 \%$ in GR after the supplementation of salicylic acid in PQ culture set as shown in Fig. 3b-e (significant at $P \leq 0.05$ ).

PQ stimulate the enzyme activity of SOD, CAT, APX GPX and GR in Azolla-Anabaena symbiotic system ${ }^{33}$ and in Chlorella vulgaris ${ }^{2}$. Accumulation of hydrogen peroxide plays an important role as signalling molecule and activates other set of enzymes i.e., CAT GPX and APX. CAT and GPX that catalyse the dismutation of hydrogen peroxide into water and oxygen. APX uses ascorbate as reducing power and potentially catalyze the removal of superoxide and hydrogen peroxide and GR accumulates GSH from GSSH and ascorbate for ascorbate peroxidase in ascorbate-glutathione cycle ${ }^{5}$. 
Salicylic acid enhanced the activity of SOD, CAT, APX GPX and GR in the present study. Salicylic acid regulates the antioxidant enzymes under stressful condition and maintain the redox state of cell (Chen et al., 2016) $)^{39}$. It is reported from higher plants that SA + PQ treatment provide the protection against PQ induced toxicity by activation of antioxidant enzymes in to barley ${ }^{36}$ and in Vigna radiate under mancozeb (fungicide), chlorpyrifos (insecticide), under metribuzin (herbicide) ${ }^{15}$ and in rice plant ${ }^{18}$ under quinclorac toxicity.

\section{Effect of PQ and SA+PQ on GSH Content and Redox State of Microchaete sp. under PQ Stress.} Glutathione is a tri-peptide non-enzymatic antioxidant compound that operates in cellular detoxification and maintain the redox homeostasis of the cell. It occurs in two form reduced glutathione (GSH) and oxidized glutathione $(\mathrm{GSSH})^{20}$. GSSH further gets reduced by glutathione reductase. This cycle regulates the redox homeostasis of the cell ${ }^{40}$. The reduced glutathione (GSH) was decreased in PQ treated culture by $25.29 \%$ as compared to control. The culture supplemented with both SA and PQ showed $67.42 \%$ increased of GSH as compared to control. The GSSH increased by $56.79 \%$ in PQ treated culture as compared to control, whereas $80.69 \%$ reduction was recorded as compared to $\mathrm{PQ}$ treated culture. The ratio of reduced glutathione to oxidized glutathione was lowered in PQ treated culture by $20.94 \%$ as compared to control and increased in $\mathrm{SA}+\mathrm{PQ}$ culture by $28.02 \%$ as compared to $\mathrm{PQ}$ treated culture (Fig. 4a-c). The activity of glutathione reductase depends on NADPH, and PQ blocks the regeneration of $\mathrm{NADPH}$ from NADP ${ }^{+}$which may be the reason of reduced GR activity which in turn reduced its activity and destabilizes the redox state. $\mathrm{PQ}$ has been also known to reduce the glutathione in cucumber ${ }^{41}$ and barley $^{3}$. Treatment of SA showed protective effect and increased the GSH content in barley under PQ stress $^{3}$ and watermelons under excess boron ${ }^{42}$.

Effect of $P Q$ and $S A+P Q$ on enhancement of the antioxidant and antibacterial activity of Microchaete sp. DPPH assay was performed to monitor the fluctuation in the antioxidant activity in all cultures. The antioxidant capacity of all sets was evaluated by monitoring DPPH-radical scavenging activity (Fig. 5a). Methanolic extract of all cultures showed improved scavenging effect that indicated the production of the antioxidant compound. DPPH scavenging activity (\%) was recorded as $92.11 \pm 2.76$ in control, 85.97 in $P Q$ treated culture, $73.69 \pm 1.90$ salicylic acid-treated culture, and $61.80 \pm 2.60$ in those culture set that contain both $S A$ and $P Q$ (significant at $P \leq 0.05$ ). Moreover, the increased phenols contents in presence of $S A+P Q$ may also be responsible for higher antioxidant activity. The scavenging activity of phenols depends on the losing of a proton from phenolic compound to bounding with DPPH radical ${ }^{43}$. Studies on abiotic stress have also shown that salicylic acid manages the oxidative stress by enhancing the antioxidant enzyme activity for example in rosemary ${ }^{44}$, in mung bean, alfalfa tomato and lemon balm under salinity, 46, 47,48, in Phaseolus aureus and flex plant under cadmium stress ${ }^{49,50}$.

The antibacterial activity was evaluated by using aqueous extracts of each culture against Pseudomomas aeruginosa. The diameters of the zone of inhibition was recorded in millimetres (Fig. 5b). Control sample showed no zone of inhibition but PQ treated samples (60 and $80 \mu \mathrm{g} / \mathrm{ml}$ ) exhibited zone of inhibition (9.6 mm and $11 \mathrm{~mm}$ diameter). SA supplementation in PQ exposed culture showed zone of 
inhibition in all tested concentration of extract $(20,40,60,80 \mu \mathrm{g} / \mathrm{ml})$ that increased in a dose dependent manner $(10 \mathrm{~mm}, 10.4 \mathrm{~mm}, 16.5 \mathrm{~mm}$ and $20 \mathrm{~mm})$. Zone of inhibition by streptomycin was recorded as $10.5 \mathrm{~mm}, 10.8 \mathrm{~mm}, 20 \mathrm{~mm}$ and $20.2 \mathrm{~mm}$ from lower to higher concentration respectively. Thus, the best anti-bacterial activity was found in presence of $S A+P Q$. Treatment of salicylic acid in rosemary also showed antibacterial activity under salinity. The enhanced phenolic and endogenous IAA and SA content and antioxidant compound by salicylic acid supplementation may be the reason for the enhancement of antioxidant and antibacterial activity. In this study, exogenous application of salicylic acid to PQ exposed cultures alters the antioxidant activities. The antioxidant and antibacterial activity results showed that SA supplementation not only resist abiotic stress but also provide resilience against biotic stress. These scavenging activities showed that SA modulates antioxidant activity. The antioxidant and antibacterial activity showed the tolerance ability of Microchaete against PQ after SA supplementation.

\section{Effect of $P Q$ and $S A+P Q$ on phenolic compound and phytohormone of Microchaete sp. under PQ stress.} Accumulation of phenolic compounds was found in all cultures. As compared to control, PQ-treated culture showed an $8.85 \%$ increase in total phenolic content (significant at $P \leq 0.05$ ). Supplementation of salicylic acid showed a $29.07 \%$ increase in total phenolic content as compared to PQ exposed culture (significant at $P \leq 0.05$ ). SA induced the production of phenolic compound in culture with both salicylic acid and PQ as observed in our study (Fig. 6a). Due to different environmental stress, plant produces secondary metabolites such as phenolic compounds, terpenoids, flavonoids, etc. for protection. These metabolites have high antioxidant potential and play a major role in defense against stress. Radwan. $(2012)^{17}$, reported that salicylic acid induced the phenolic compound in clethodium herbicide stressed maize to enable it to survive.

The endogenous phytohormones (IAA \& SA) were also induced in all sets (Fig. 6b-c). The endogenous level of IAA increased by $13.43 \%$ in $P Q$ as compared to control, whereas the supplementation of SA in PQ exposed culture resulted in further increase by $2.96 \%$ only as compared to PQ treated cultures. The amount of endogenous salicylic acid also increased in PQ treated culture by $96.59 \%$ as compared to control. The supplementation of salicylic acid with $P Q$ resulted in higher SA accumulation by $4.72 \%$ as compared to $P Q$ treated culture (Fig. 6c). Exogenous supplementation of SA in higher plants induced endogenous SA content e.g. in cucumber under salt stress ${ }^{31}$ and in Baikal skullcap under salt and drought stress ${ }^{51}$. Exogenous application of salicylic acid induced both IAA and SA in maize and cucumber under drought stress ${ }^{52,53}$.

\section{Declarations}

\section{Acknowledgements}

Authors are thankful to Indian Agriculture Research Institute (IARI), New Delhi for providing cyanobacterial strain. We are also thankful to the Departmental instrument facility (DIF-Biosciences).

\section{Declaration of competing interests}




\section{References}

1. Abinandan, S., Subashchandrabose, S. R., Venkateswarlu, K. \& Megharaj, M. Soil microalgae and cyanobacteria: the biotechnological potential in the maintenance of soil fertility and health. Critical reviews in biotechnology, 39 (8), 981-998 (2019).

2. Qian, H. et al. Inhibitory effects of paraquat on photosynthesis and the response to oxidative stress in Chlorella vulgaris. Ecotoxicology, 18 (5), 537-543 (2009).

3. Ananieva, E. A., Christov, K. N. \& Popova, L. P. Exogenous treatment with salicylic acid leads to increased antioxidant capacity in leaves of barley plants exposed to paraquat. Journal of Plant Physiology, 161 (3), 319-328 (2004).

4. Eullaffroy, P. \& Vernet, G. The F684/F735 chlorophyll fluorescence ratio: a potential tool for rapid detection and determination of herbicide phytotoxicity in algae. Water Res, 37 (9), 1983-1990 (2003).

5. Blokhina, O., Virolainen, E. \& Fagerstedt, K. V. Antioxidants, oxidative damage and oxygen deprivation stress: a review. Annals of Botany, 91 (2), 179-194 (2003).

6. Chittora, D., Meena, M., Barupal, T., Swapnil, P. \& Sharma, K. Cyanobacteria as a source of biofertilizers for sustainable agriculture. Biochemistry and biophysics reports, 22, 100737 (2020).

7. Mishra, U. \& Pabbi, S. Cyanobacteria: a potential biofertilizer for rice. Resonance, 9 (6), 6-10 (2004).

8. Enyedi, A. J., Yalpani, N., Silverman, P. \& Raskin, I. Signal molecules in systemic plant resistance to pathogens and pests. Cell, 70 (6), 879-886 (1992).

9. Rao, M. V., Paliyath, G., Ormrod, D. P., Murr, D. P. \& Watkins, C. B. Influence of salicylic acid on H2O2 production, oxidative stress, and H2O2-metabolizing enzymes (salicylic acid-mediated oxidative damage requires H2O2). Plant Physiology, 115 (1), 137-149 (1997).

10. Szepesi, Ã. et al. Salicylic acid improves acclimation to salt stress by stimulating abscisic aldehyde oxidase activity and abscisic acid accumulation, and increases $\mathrm{Na}+$ content in leaves without toxicity symptoms in Solanum lycopersicum L. Journal of Plant Physiology, 166 (9), 914-925 (2009).

11. Mishra, A. \& Choudhuri, M. A. Effects of salicylic acid on heavy metal-induced membrane deterioration mediated by lipoxygenase in rice. Biol. Plant, 42 (3), 409-415 (1999).

12. Senaratna, T., Touchell, D., Bunn, E. \& Dixon, K. Acetyl salicylic acid (Aspirin) and salicylic acid induce multiple stress tolerance in bean and tomato plants. Plant Growth Regulation, 30 (2), 157-161 (2000).

13. Clarke, S. M., Mur, L. A., Wood, J. E. \& Scott, I. M. Salicylic acid dependent signaling promotes basal thermotolerance but is not essential for acquired thermotolerance in Arabidopsis thaliana. The Plant Journal, 38 (3), 432-447 (2004). 
14. Janda, T., Szalai, G., Tari, I. \& Paldi, E. Hydroponic treatment with salicylic acid decreases the effects of chilling injury in maize (Zea mays L.) plants. Planta, 208 (2), 175-180 (1999).

15. Fatma, F., Kamal, A. \& Srivastava, A. Exogenous application of salicylic acid mitigates the toxic effect of pesticides in Vigna radiata (L.) Wilczek. Journal of plant growth regulation, 37 (4), 1185-1194 (2018).

16. Cui, J., Zhang, R., Wu, G. L., Zhu, H. M. \& Yang, H. Salicylic acid reduces napropamide toxicity by preventing its accumulation in rapeseed (Brassica napus L.). Archives of environmental contamination and toxicology, 59 (1), 100-108 (2010).

17. Radwan, D. E. M. Salicylic acid induced alleviation of oxidative stress caused by clethodim in maize (Zea mays L.) leaves. Pesticide biochemistry and physiology, 102 (2), 182-188 (2012).

18. Wang, J. et al. Salicylic acid mediates antioxidant defense system and ABA pathway related gene expression in Oryza sativa against quinclorac toxicity. Ecotoxicology and Environmental Safety, 133, 146-156 (2016).

19. Nazar, R., Umar, S., Khan, N. A. \& Sareer, O. Salicylic acid supplementation improves photosynthesis and growth in mustard through changes in proline accumulation and ethylene formation under drought stress. South African Journal of Botany, 98, 84-94 (2015).

20. Noctor, G., Gomez, L., Vanacker, H. \& Foyer, C. H. Interactions between biosynthesis, compartmentation and transport in the control of glutathione homeostasis and signalling. Journal of experimental botany, 53 (372), 1283-1304 (2002).

21. Li, G., Peng, X., Wei, L. \& Kang, G. Salicylic acid increases the contents of glutathione and ascorbate and temporally regulates the related gene expression in salt-stressed wheat seedlings. Gene, $529(2)$, $321-325$ (2013).

22. Pimentel, D. \& Levitan, L. Pesticides: amounts applied and amounts reaching pests. Bioscience, 36 (2), 86-91 (1986).

23. Zhang, H. et al. Effects of paraquat on the growth of cyanobacteria and green algae. Acta Sci. Circum, 33, 1441-1445 (2013).

24. Thajamanbi, M., Rout, J. \& Thajuddin, N. Blue green algae from rice fields of Karimganj district, Assam, North East India. Life, 50, 23 (2016).

25. Tansai, S., Issakul, K. \& Ngearnpat, N. Toxicity of paraquat on growth of cyanobacteria (Nostoc sp. N1 and Anabaena sp. A1) and germination of rice seed (san-pah-twang 1). The 6th International Conference on Biochemistry and Molecular Biology. Thailand(2018).

26. Dragolova, D., Chaneva, G., Gemishev, T. \& Vassilev, G. Physiological changes in the cyanobacteria Anabaena variabilis and Plectonema boryanum caused by paraquat toxicity. Cr Acad Bulg Sci, 55 (5), 5-85 (2002).

27. Pandey, F. K., Kumar, S. \& Bhatnagar, T. Toxic effect of an herbicide on growth and nitrogen formation of two N2-fixing cyanobacteria. Intr J Curr Res, 3, 14-16 (2011).

28. Zhang, W. et al. Effects of paraquat on photosynthetic pigments, antioxidant enzymes, and gene expression in Chlorella pyrenoidosa under mixotrophic compared with autotrophic conditions. Arch. 
Environ. Contam. Toxicol, 67 (4), 593-600 (2014).

29. Czerpak, R., Bajguz, A., Gromek, M., Kozłowska, G. \& Nowak, I. Activity of salicylic acid on the growth and biochemism of Chlorella vulgaris Beijerinck. Acta Physiologiae Plantarum, 24 (1), 45 (2002).

30. Aeby, G. S. et al. First record of black band disease in the Hawaiian archipelago: response, outbreak status, virulence, and a method of treatment. PLoS One, 10 (3), e0120853 (2015).

31. Kim, Y., Kim, S. \& Shim, I. S. Exogenous salicylic acid alleviates salt-stress damage in cucumber under moderate nitrogen conditions by controlling endogenous salicylic acid levels. Horticulture, Environment, and Biotechnology, 58 (3), 247-253 (2017).

32. Elstner, E. F., Wagner, G. A. \& Schutz, W. Activated oxygen in green plants in relation to stress situations. In Current topics in plant biochemistry and physiology: Proceedings of the Plant Biochemistry and Physiology Symposium held at the University of Missouri, Columbia (USA)(1988).

33. Sood, A., Pabbi, S. \& Uniyal, P. L. Effects of paraquat on lipid peroxidation and antioxidant enzymes in aquatic fern Azolla microphylla. Russian Journal of Plant Physiology, 58 (4), 667-673 (2011).

34. Wu, L. et al. Physiological effects of the herbicide glyphosate on the cyanobacterium Microcystis aeruginosa. Aquatic Toxicology, 178, 72-79 (2016).

35. Galhano, V., Peixoto, F. \& Gomes-Laranjo, J. Bentazon triggers the promotion of oxidative damage in the Portuguese rice field cyanobacterium Anabaena cylindrica: response of the antioxidant system. Environmental toxicology, 25 (5), 517-526 (2010).

36. Ananieva, E. A., Alexieva, V. S. \& Popova, L. P. Treatment with salicylic acid decreases the effects of paraquat on photosynthesis. Journal of Plant Physiology, 159 (7), 685-693 (2002).

37. Radwan, D. E. M., Mohamed, A. K., Fayez, K. A. \& Abdelrahman, A. M. Oxidative stress caused by Basagran ${ }^{\circledR}$ herbicide is altered by salicylic acid treatments in peanut plants. Heliyon, 5 (5), e01791 (2019).

38. Wang, Y. S. et al. Salicylic acid modulates aluminum-induced oxidative stress in roots of Cassia tora. Acta Botanica Sinica-English Edition, 46 (7), 819-828 (2004).

39. Chen, Y. E. et al. Effect of salicylic acid on the antioxidant system and photosystem II in wheat seedlings. Biologia Plantarum, 60 (1), 139-147 (2016).

40. Cameron, J. C. \& Pakrasi, H. B. Essential role of glutathione in acclimation to environmental and redox perturbations in the cyanobacterium Synechocystis sp. PCC 6803. Plant physiology, 154 (4), 1672-1685 (2010).

41. Lin, S. H., Liu, Z. J., Xu, P. L., Fang, Y. Y. \& Bai, J. G. Paraquat pre-treatment increases activities of antioxidant enzymes and reduces lipid peroxidation in salt-stressed cucumber leaves. Acta physiologiae plantarum, 33 (2), 295-304 (2011).

42. Moustafa-Farag, M. et al. Salicylic acid stimulates antioxidant defense and osmolyte metabolism to alleviate oxidative stress in watermelons under excess boron. Plants, 9 (6), 724 (2020).

43. Viljanen, K. Protein oxidation and protein-lipid interactions in different food models in the presence of berry phenolics (2005). 
44. El-Esawi, M. A. et al. Salicylic acid-regulated antioxidant mechanisms and gene expression enhance rosemary performance under saline conditions. Frontiers in Physiology, 8, 716 (2017).

45. Khan, N., Syeed, S., Masood, A., Nazar, R. \& Iqbal, N. Application of salicylic acid increases contents of nutrients and antioxidative metabolism in mungbean and alleviates adverse effects of salinity stress. International Journal of Plant Biology, 1 (1), e1-e1 (2010).

46. Palma, F., Lopez-Gomez, M., Tejera, N. A. \& Lluch, C. Salicylic acid improves the salinity tolerance of Medicago sativa in symbiosis with Sinorhizobium meliloti by preventing nitrogen fixation inhibition. Plant science, 208, 75-82 (2013).

47. Szepesi, A. Influence of exogenous salicylic acid on antioxidant enzyme activities in the roots of salt stressed tomato plants. Acta Biologica Szegediensis, 52 (1), 199-200 (2008).

48. Safari, F., Akramian, M. \& Salehi-Arjmand, H. Physiochemical and molecular responses of saltstressed lemon balm (Melissa officinalis L.) to exogenous protectants. Acta Physiologiae Plantarum, 42(2), 1-10 (2020).

49. Zhang, F. et al. Exogenous application of salicylic acid alleviates cadmium toxicity and reduces hydrogen peroxide accumulation in root apoplasts of Phaseolus aureus and Vicia sativa. Plant cell reports, 30 (8), 1475-1483 (2011).

50. Belkadhi, A. et al. Salicylic acid increases tolerance to oxidative stress induced by hydrogen peroxide accumulation in leaves of cadmium-exposed flax (Linum usitatissimum L.). Journal of Plant Interactions, 9 (1), 647-654 (2014).

51. Su, H. et al. Endogenous salicylic acid shows different correlation with baicalin and baicalein in the medicinal plant Scutellaria baicalensis Georgi subjected to stress and exogenous salicylic acid. PloS one, 13 (2), e0192114 (2018).

52. El-Mergawi, R. A. \& Abd El-Wahed, M. S. Effect of exogenous salicylic acid or indole acetic acid on their endogenous levels, germination, and growth in maize. Bulletin of the National Research Centre, 44 (1), 1-8 (2020).

53. Dong, C. J., Liu, X. Y., Xie, L. L., Wang, L. L. \& Shang, Q. M. Salicylic acid regulates adventitious root formation via competitive inhibition of the auxin conjugation enzyme CsGH3. 5 in cucumber hypocotyls. Planta, 252 (5), 1-15 (2020).

54. Stanier, R. Y., Kunisawa, R., Mandel, M. C. B. G. \& Cohen-Bazire, G. Purification and properties of unicellular blue-green algae (order Chroococcales). Bacteriological reviews, 35 (2), 171-205 (1971).

55. Mackinney, G. Absorption of light by chlorophyll solutions. J. Biol. Chem, 140 (2)), 315-322 (1941).

56. Singh, A. \& Zahra, K. LC50 assessment of cypermethrin in Heteropneustes fossilis: Probit analysis. Int J Fisheries Aquatic Studies, 5 (5), 126-130 (2017).

57. Naaz, H. et al. Exogenous salicylic acid mediated herbicide (Paraquat) resistance in cyanobacterial biofertilizer Microchaete sp. NCCU-342. Environmental Science and Pollution Research.1-11(2021).

58. Singleton, V. L. \& Rossi, J. A. Colorimetry of total phenolics with phosphomolybdic phosphotungstic acid reagents. Am. J. Enol. Vitic, 16, 144-158 (1965). 
59. Ehmann, A. The Van Urk-Salkowski reagent-a sensitive and specific chromogenic reagent for silica gel thin-layer chromatographic detection and identification of indole derivatives. Journal of Chromatography A, 132 (2)), 267-276 (1977).

60. Raskin, I., Turner, I. M. \& Melander, W. R. Regulation of heat production in the inflorescences of an Arum lily by endogenous salicylic acid. Proceedings of the National Academy of Sciences. 86(7), 2214-2218(1989).

61. Warrier, R. R., Paul, M. \& Vineetha, M. V. Estimation of salicylic acid in Eucalyptus leaves using spectrophotometric methods. Genetics and plant physiology, 3 (1-2), 90-97 (2013).

62. Anderson, M. E. Determination of glutathione and glutathione disulfide in biological samples. Methods in enzymology, 113, 548-555 (1985).

63. Asgher, M., Khan, N. A., Khan, M. I. R., Fatma, M. \& Masood, A. Ethylene production is associated with alleviation of cadmium-induced oxidative stress by sulfur in mustard types differing in ethylene sensitivity. Ecotoxicology and environmental safety, 106, 54-61 (2014).

64. Elia, A. C., Galarini, R., Taticchi, M. I., Dörr, A. J. M. \& Mantilacci, L. Antioxidant responses and bioaccumulation in Ictalurus melas under mercury exposure. Ecotoxicology and Environmental Safety, 55 (2), 162-167 (2003).

65. Shimada, K., Fujikawa, K., Yahara, K. \& Nakamura, T. Antioxidative properties of xanthan on the autoxidation of soybean oil in cyclodextrin emulsion. Journal of agricultural and food chemistry, 40 (6), 945-948 (1992).

66. Matson, J. M., Kopeck, M. J. H. \& Quiet, P. G. Evaluation of the Bauer Kirby-Sherris-Turck single-disc diffusion method of antibiotic susceptibility testing. Antimicrobial agents and chemotherapy(1970).

67. Sole, A., Diestra, E. \& Esteve, I. Confocal laser scanning microscopy image analysis for cyanobacterial biomass determined at microscale level in different microbial mats. Microbial ecology, 57 (4), 649-656 (2009).

\section{Figures}


a.

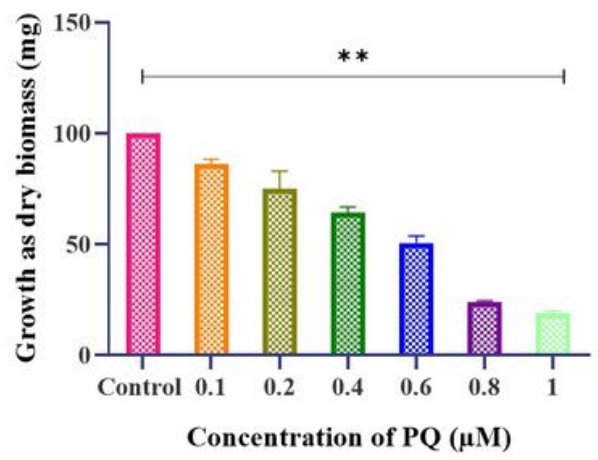

b.

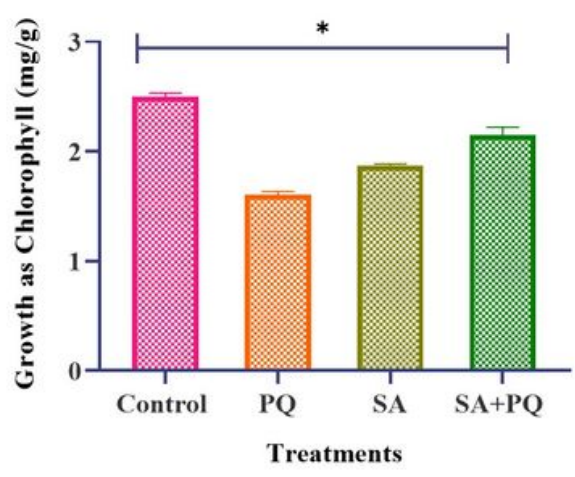

Quantitative measurement of growth c.

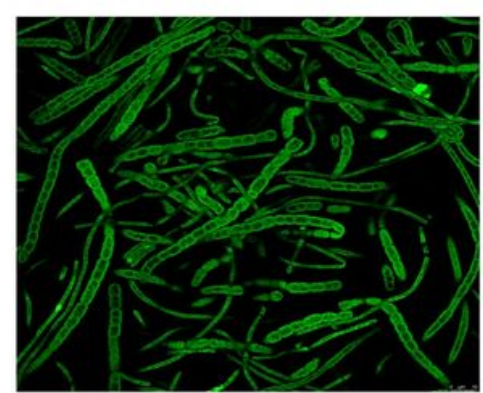

e.

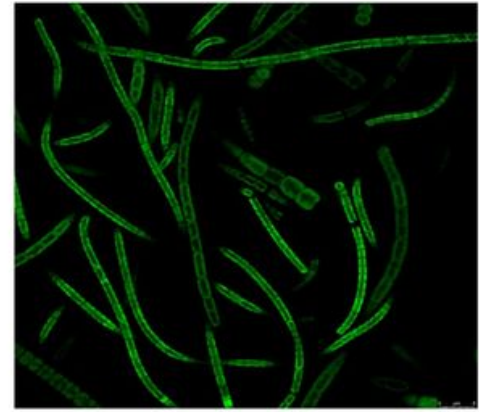

d.

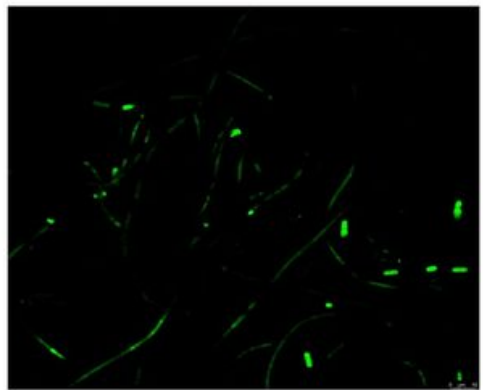

f.

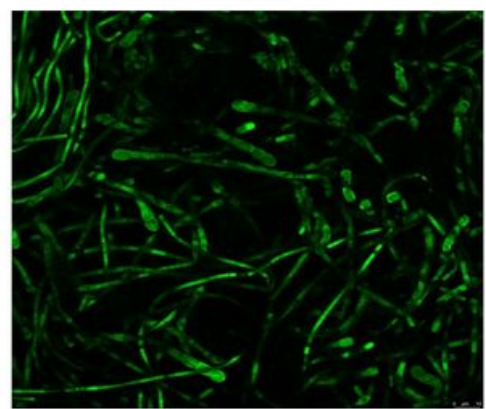

Qualitative measurement of growth through confocal imaging

\section{Figure 1}

a) Growth of Microchaete sp. under different concentration of $P Q$ b) and with salicylic acid, the values are means of three replicates \pm standard deviation (SD). Asterisk showed level of significance at $P<0.05$. Quantitative measurement of growth through confocal imaging c) control, d) PQ, e) $S A, f) S A+P Q$. 
a.

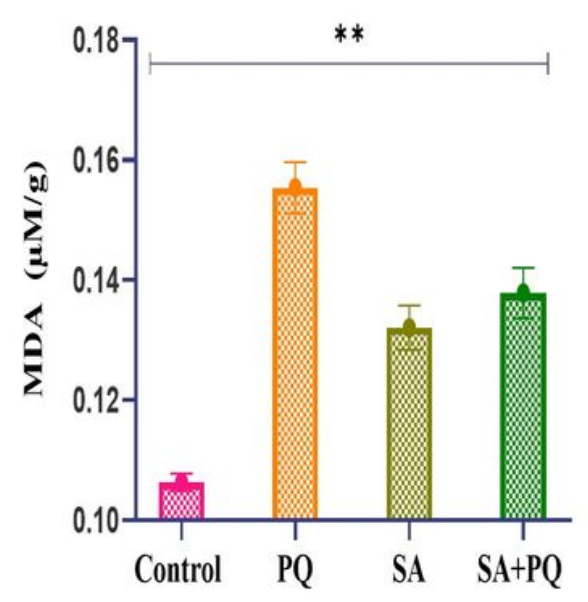

b.

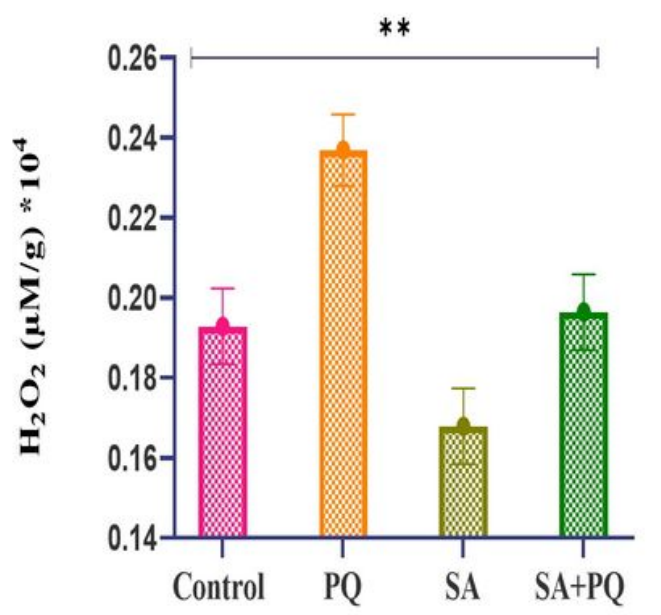

Figure 2

Effect of PQ $(0.6 \mu \mathrm{M}), \mathrm{SA}(0.3 \mathrm{mM})$ and SA+PQ on a) MDA $(\mu \mathrm{M} / \mathrm{g})$ and b) $\mathrm{H} 2 \mathrm{O} 2(\mu \mathrm{M} / \mathrm{g})$ content of Microchaete sp. NCCU-342. The values are means of three replicates \pm standard deviation (SD). Asterisk showed level of significance at $P<0.05$. 

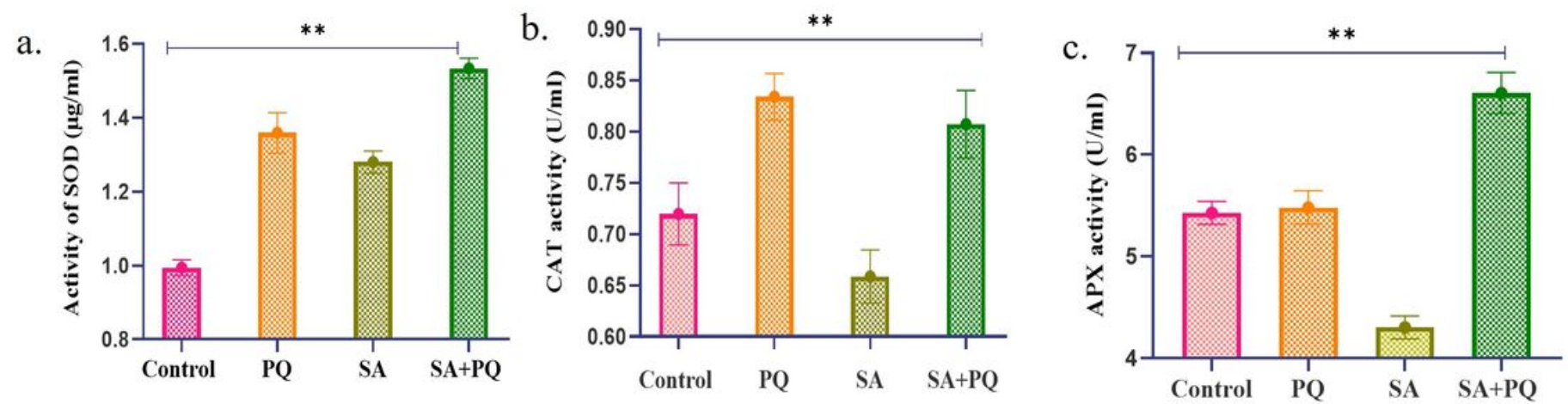

d.

e.
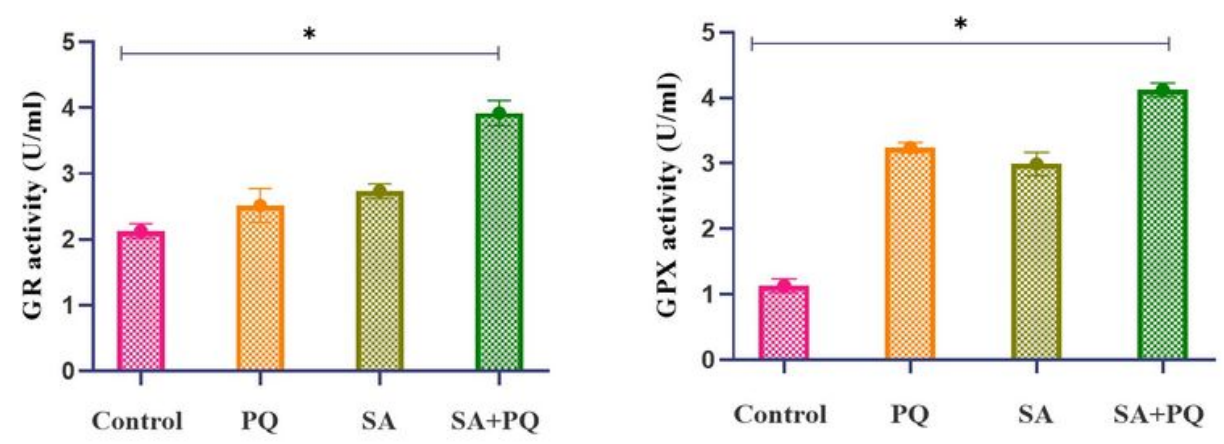

Figure 3

Effect of PQ $(0.6 \mu \mathrm{M}), \mathrm{SA}(0.3 \mathrm{mM})$ and $\mathrm{SA}+\mathrm{PQ}$ on antioxidant enzymes activity of a) SOD, b) CAT and c) APX d) GR, e) GPX of Microchaete sp. NCCU-342. The values are means of three replicates \pm standard deviation (SD). Asterisk showed level of significance at $P<0.05$. 
a.

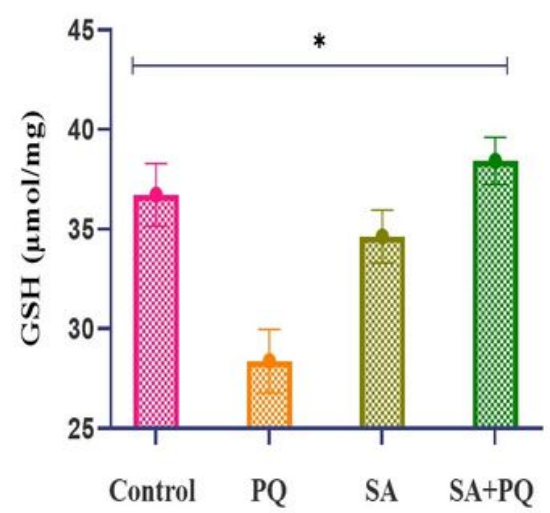

b.

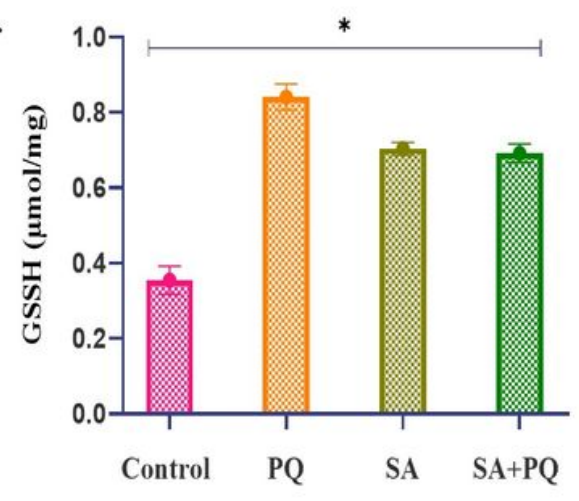

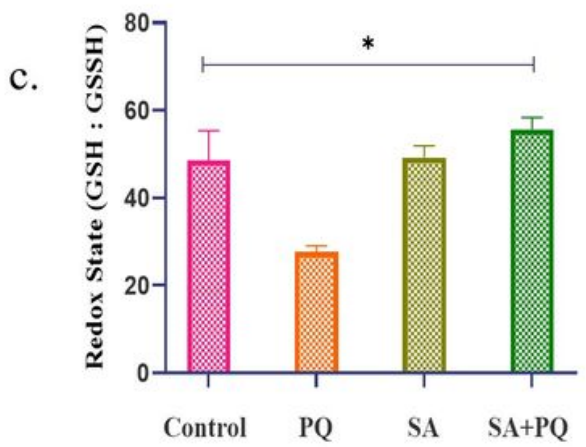

Figure 4

Effect of $P Q(0.6 \mu M), S A(0.3 \mathrm{mM})$ and $S A+P Q$ on glutathione content $a)$ reduced and $b)$ oxidized glutathione and c) redox stateof Microchaete sp. NCCU-342. The values are means of three replicates \pm standard deviation (SD). Asterisk showed level of significance at $P<0.05$. 
a.

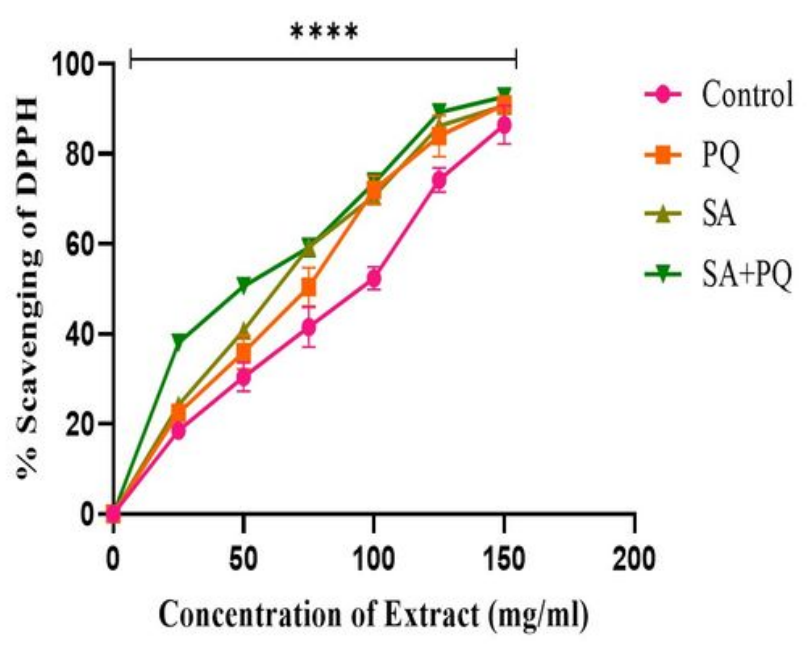

b.

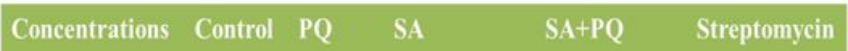

\begin{tabular}{|c|c|c|c|c|c|}
\hline $20 \mu \mathrm{g} / \mathrm{ml}$ & No ZOI & No ZOI & No ZOI & $\begin{array}{l}10.33 \pm 0.054 \\
\mathrm{~mm}\end{array}$ & $\begin{array}{l}10.46 \pm 0.05 \\
\mathrm{~mm}\end{array}$ \\
\hline $40 \mu \mathrm{g} / \mathrm{ml}$ & No ZOI & No ZOI & $\begin{array}{l}7.03 \pm 0.03 \\
\mathrm{~mm}\end{array}$ & $\begin{array}{l}10.36 \pm 0.06 \\
\mathrm{~mm}\end{array}$ & $\begin{array}{l}10.86 \pm 0.002 \\
\mathrm{~mm}\end{array}$ \\
\hline $60 \mu \mathrm{g} / \mathrm{ml}$ & No ZOI & $\begin{array}{l}7.05 \pm 0 \\
06 \mathrm{~mm}\end{array}$ & $\begin{array}{l}9.03 \pm 0.05 \\
\mathrm{~mm}\end{array}$ & $\begin{array}{l}16.46 \pm 0.057 \\
\mathrm{~mm}\end{array}$ & $\begin{array}{l}20.06 \pm 0.054 \\
\mathrm{~mm}\end{array}$ \\
\hline
\end{tabular}

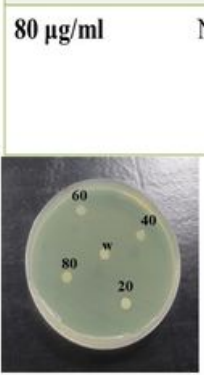

Control

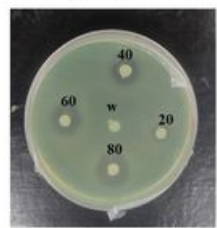

SA

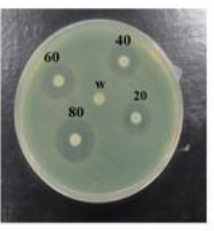

Streptomycin

\section{Figure 5}

Effect of PQ $(0.6 \mu \mathrm{M}), \mathrm{SA}(0.3 \mathrm{mM})$ and SA+PQ on a) antioxidant activity and b) antibacterial activity of Microchaete sp. NCCU-342. The values are means of three replicates \pm standard deviation (SD). Asterisk showed level of significance at $P<0.05$. 
a.

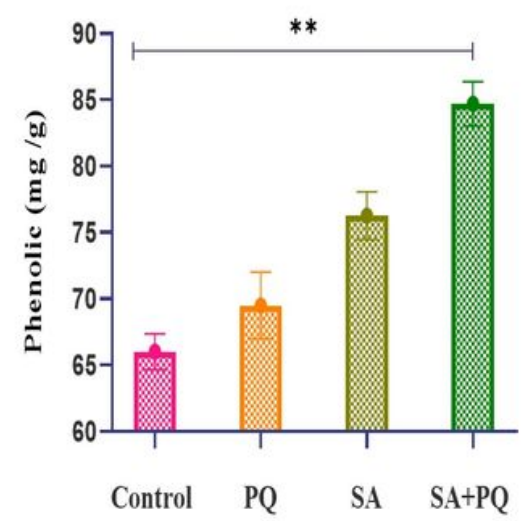

b.

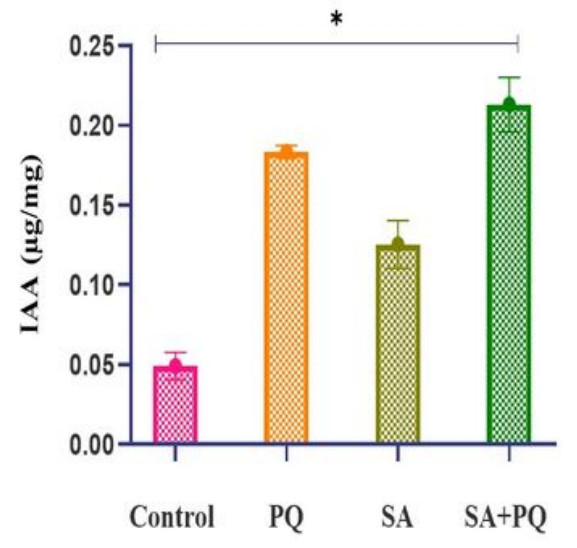

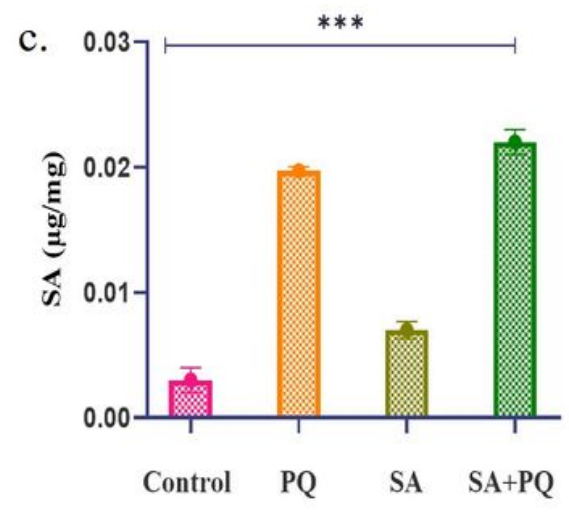

\section{Figure 6}

Effect of PQ $(0.6 \mu \mathrm{M}), \mathrm{SA}(0.3 \mathrm{mM})$ and SA+PQ on a) Phenolic $(\mathrm{mg} / \mathrm{g}), \mathrm{b}) \mathrm{IAA}(\mu \mathrm{g} / \mathrm{mg})$ and c) SA $(\mu \mathrm{g} / \mathrm{mg})$ content of Microchaete sp. NCCU-342. The values are means of three replicates \pm standard deviation (SD). Asterisk showed level of significance at $P<0.05$. 